\title{
Jigless knotless internal brace technique for acute Achilles tendon rupture: a case series study
}

\author{
Po-Yen Ko ${ }^{1,2,3}$, Ming-Tung Huang ${ }^{3}$, Chia-Lung Li, ${ }^{2,3}$, Wei-Ren Su ${ }^{3,4}$, I-Ming Jou ${ }^{5,6}$ and Po-Ting $\mathrm{Wu}^{1,2,3,4,7^{*}}$
}

\begin{abstract}
Purpose: To mitigate the risk of poor wound healing and of infection associated with the open repair of Achilles tendon midsubstance ruptures, minimally invasive techniques have been developed. We report our preliminary results after reviewing our "jigless knotless internal brace technique."

Methods: Patients were placed in prone position and a transverse $3-\mathrm{cm}$ incision was made proximal to the palpable ruptured end. The proximal ruptured end was pulled out, gently debrided, and sutured using Krackow locking loops. Percutaneous sutures were crisscrossed through the distal tendon stump and looped around the Krackow sutures over the proximal stump. The ipsilateral Krackow sutures and the contralateral crisscrossed sutures were subcutaneously passed through two mini-incisions over the posterior calcaneus tuberosity and seated at the tuberosity with two 4.5-mm knotless suture anchors. All patients underwent the same post-operative rehabilitation protocol and regular follow-ups for at least 1 year.
\end{abstract}

Results: We recruited 10 patients (mean age, 37.3 years) who scored 100 points on the American Orthopaedic Foot and Ankle Society (AOFAS) scale, and who returned to their preoperative exercise levels 1-year postoperatively with no complications.

Conclusion: Our method is simple, effective, and requires no special tools. It might be a reliable option for Achilles tendon repair.

Level of evidence: III

Keywords: Achilles tendon rupture, Minimally invasive, Knotless, Internal brace

\section{Introduction}

Although the Achilles tendon is the human body's strongest tendon, it is also the tendon most frequently ruptured [1]. Over the past quarter-century, the rupture incidence rate has significantly increased [2-4]. A Danish national study [3] reported more than 33,000 ruptures (males 47/100,000; females 17/100,000).

Achilles tendon management options have been reported: non-operative management with a short leg-cast, a brace in an equinus position, and surgical repair [4-6].

\footnotetext{
* Correspondence: anotherme500@gmail.com

'Department of Biomedical Engineering, National Cheng Kung University, Tainan, Taiwan

${ }^{2}$ Department of Orthopedics, National Cheng Kung University Hospital Dou-Liu Branch, College of Medicine, National Cheng Kung University, Yunlin, Taiwan

Full list of author information is available at the end of the article
}

There was no consensus on the best option [7-10]. Some studies have reported lower rerupture rates in operative groups $[11,12]$, but others have claimed nearly identical rerupture rates in both operative and nonoperative groups [13]. For active young athletes who must quickly return to competition, surgery is indicated to avoid muscle atrophy after non-operative management [14]. There are several operative Achilles repair methods: percutaneous and minimally invasive surgery (MIS), and open repair with or without augmentation. One high-quality meta-analysis [15] reported no significant differences in deep infection, rerupture, tissue adhesion, or nerve injury rates between minimally invasive surgery (MIS) and open surgery; MIS, however, has better subjective outcomes and a significantly lower

(c) The Author(s). 2019 Open Access This article is distributed under the terms of the Creative Commons Attribution 4.0 International License (http://creativecommons.org/licenses/by/4.0/), which permits unrestricted use, distribution, and 
superficial infection rate [15]. Despite these benefits, injuring the sural nerve during MIS is still a risk [16-18].

A recent cadaver study reported that the sural nerve would crosses the lateral border of the Achilles tendon 8to $10-\mathrm{cm}$ proximal to the superior border of the calcaneal tuberosity in most cases [19]. Therefore, a surgeon can avoid injuring the sural nerve by doing all percutaneous procedures within $8 \mathrm{~cm}$ proximal to the calcaneal tuberosity. Hence, we have developed a novel Achilles tendon surgical method called the "jigless knotless internal brace technique" to repair the Achilles tendon. We report our preliminary results using this technique.

\section{Materials and methods \\ Patients}

Written informed consent was obtained from all patients. All procedures were approved by the National Cheng Kung University Hospital's (NCKUH) Institutional Review Board. Consecutive patients treated for acute Achilles tendon injury at NCKUH between January 2015 and July 2017were evaluated. Our inclusion criteria were a positive Simmonds test (aka Thompson test or SimmondsThompson test) and a palpable defect in the Achilles tendon corresponding to a midsubstance rupture. Surgery occurred within 2 weeks post-injury. The exclusion criteria were a cutting or penetrating injury, an injury more than 2 weeks old, a neurological or a psychiatric disorder, pregnancy, being less than 18 years old, an incomplete medical record, an inadequate follow-up, autoimmune or connective tissue diseases (e.g., rheumatoid arthritis), radiotherapy or chemotherapy, morbid obesity, and previous Achilles tendon surgery. Finally, 10 patients (men, 9; women, 1; minimum follow-up, 1 year; mean age, 37.3 years; age range, 20-53 years; mean body mass index [BMI], 24.5; BMI range, 22.1-29.7) were enrolled. All enrolled patients had undergone the same post-operative rehabilitation protocol, and all returned to our hospital for clinical follow-ups 2, 4, 6, and 8 weeks, and 3, 4, 6, and 12 months post-surgery.

\section{Surgical technique}

After a patient had been spinally anesthetized, they were put on the table in the prone position, and an air tourniquet was placed on the thigh. The tendon gap was palpated to identify the ruptured end. A 3-cm long transverse incision was made $2 \mathrm{~cm}$ proximal to the ruptured end. The proximal stump was gently pulled out through the transverse incision with the knee in flexion position after the stump had been freed from the surrounding paratenon and plantaris tendon, if it was present, using a 1-inch ribbon malleable retractor. The hematoma was completely debrided. Krackow locking loops were used on both sides of the soleus muscle and healthy tendon of the proximal stump ( $\mathrm{Hi}-\mathrm{Fi}^{\ominus}$ Suture;
CONMED Corporate Headquarters, Utica, NY, USA) (Fig. 1a, d). An Allis clamp (forceps) was subcutaneously inserted through the transverse incision in maximum ankle plantar flexion to maintain the tension of the distal stump, and then the percutaneous suture was crisscrossed through the distal stump (CONMED) (Fig. 1b, d). The end of the distal stump suture was subcutaneously passed through the transverse incision (Fig. 1c) and then looped through the proximal stump Krackow locking loop as the pulley (Fig. 2a, d). Two 0.5-cm long vertical incisions were bilaterally made on the posterior calcaneal tuberosity, and then the bird-beak arthroscopic suture passer was subcutaneously passed from the vertical incision to the transverse incision (Fig. 2b, d). The subcutaneous tunnel must be empty to avoid skin dimpling in the subsequent suture passage. The ipsilateral Krackow suture end and contralateral crisscross suture end were passed down to the distal mini-vertical incision (Fig. 2c). The sutures were seated at the posterior calcaneal tuberosity with two 4.5-mm suture anchors (PopLok ${ }^{\ominus}$ Knotless Suture Anchors; CONMED) (Fig. 3a, d). The tendon rupture gap became smooth and impalpable when the sutures were pulled to symmetrically proper tension in $30^{\circ}$ of knee flexion and ankle plantar flexion. The anchors were then locked. A 3-0 absorbable running stitch (Monocryl; Ethicon, Johnson \& Johnson Medical N.V., Belgium) was used at the epitenon (Fig. 3b). The wound was irrigated and closed layer by layer with 30 and 4-0 monocryl subcutaneous sutures and finally closed with reinforced antimicrobial skin closures (SteriStrips; 3 M Health Care, St. Paul, MN, USA). The Achilles tendon tension was checked with the leg erect immediately post-surgery (Fig. 3c).

\section{Rehabilitation protocol}

Patients were advised to do an active ankle-pumping exercise, to do a non-weight-bearing range of motion exercise for at least $1 \mathrm{~h}$ a day, and to walk without a cast or splint protection, all immediately post-surgery. They were also advised to walk full weight bearing (FWB) with crutches and wearing shoes with an added heel wedge (3 $\mathrm{cm})$. One-week post-surgery, patients were allowed to begin walking without ambulatory aids (canes, crutches, walkers, etc.). Two weeks post-surgery, we recommended that they reduce the added heel wedge height by $1 \mathrm{~cm}$ per week. Muscle power training with a concurrent heel-raising exercise began 1 month post-surgery, and 6 weeks later, patients were permitted to return to exercise as tolerable.

\section{Results}

Surgery-related data

The mean time between injury and surgery was 4.3 days (range, 1-9 days). The mean surgery duration was $22 \mathrm{~min}$ 

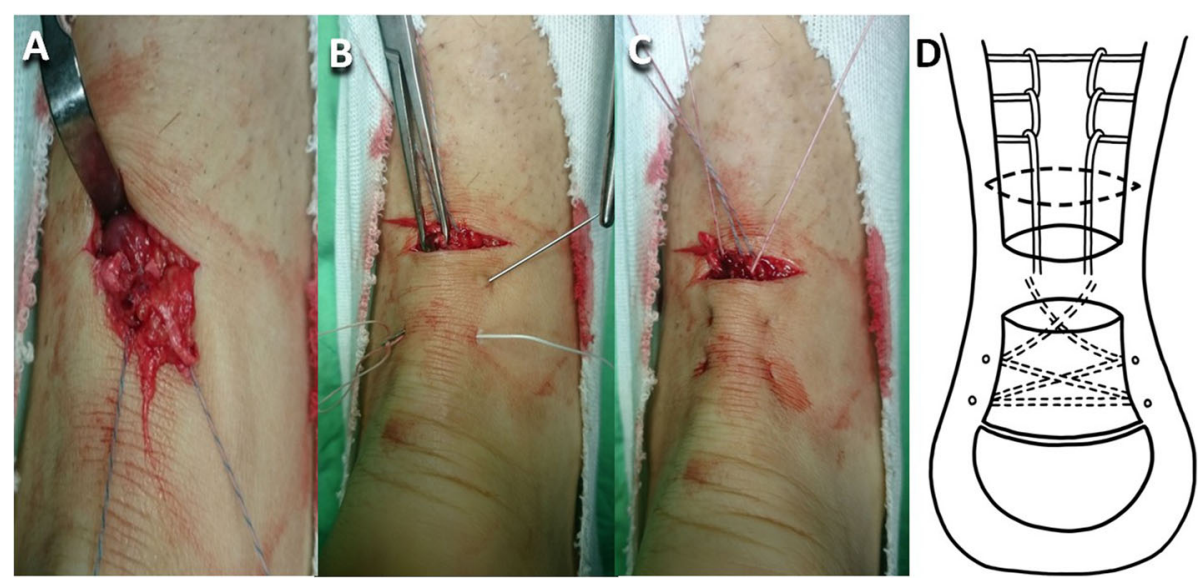

Fig. 1 a Krackow locking loop sutures were applied at the proximal stump through a $3-\mathrm{cm}$ transverse incision $2 \mathrm{~cm}$ proximal to the palpable tendon rupture gap. $\mathbf{b}$ The percutaneous suture was crisscrossed through the distal stump. $\mathbf{c}$ The end of the distal stump suture was subcutaneously passed through the transverse incision. $\mathbf{d}$ Illustration of (a)-(c)

(range, 18-36 $\mathrm{min}$ ). The transverse incision averaged 6.3 $\mathrm{cm}$ (range, $5.5-7 \mathrm{~cm}$ ) proximal to the calcaneal tuberosity.

\section{Complications}

There were no serious complications in the present study. All incisions healed well and without scar adhesions or superficial or deep infections (Fig. 4a). There were no sural nerve injuries, reruptures, deep vein thromboses, or pulmonary embolisms.

\section{Functional outcomes}

After 1 post-operative year, all 10 patients scored 100 points on the American Orthopaedic Foot and Ankle Society (AOFAS) scale, and they returned to their preoperative exercise levels. Patients needed a mean of 7.5 days (range, 3-11 days) to walk FWB without crutches and they needed a mean of 24.5 days (range, 21-28 days) to walk with a full ankle-joint range of motion (Fig. 4b, c). To stand with a raised heel without an aid, they needed an average of 35 days (range, 28-42 days) (Fig. 4d). All patients returned to their jobs, which needed an average of 45.5 days (range, 35-63 days), and to their previous athletic exercise level, which needed a mean of 147.5 days (range, 84-210 days).

\section{Discussion}

There is still no consensus about the best way to manage acute Achilles tendon ruptures [7-10]. In active young athletes who want to quickly return to competition, surgery is indicated to avoid muscle atrophy after nonsurgical treatments [14]. Because of improvements in surgical techniques, multiple MIS methods have been

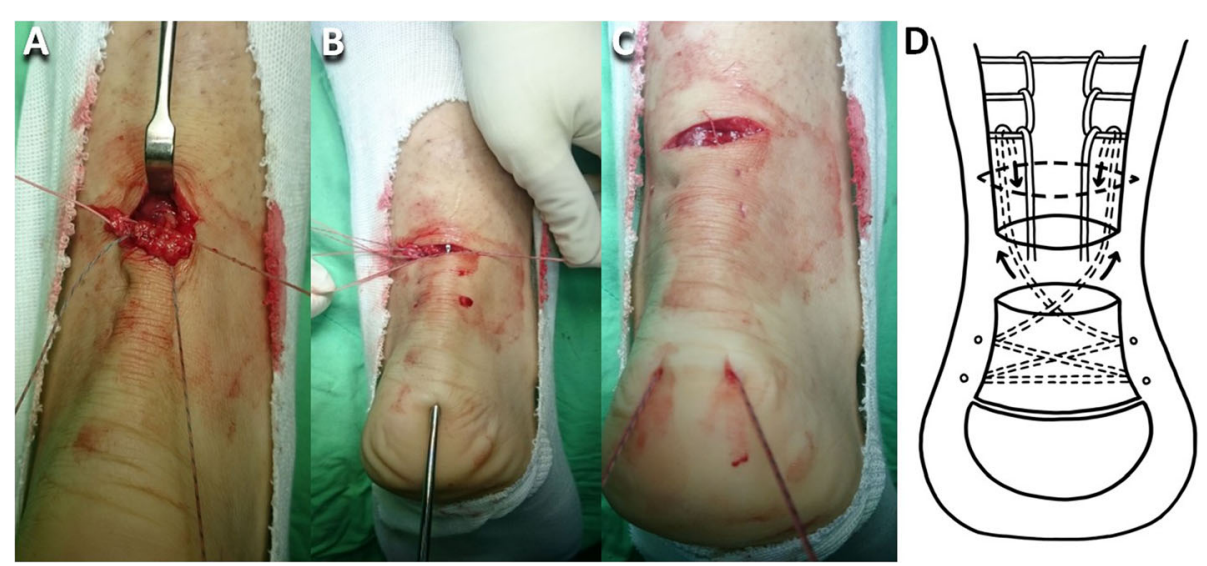

Fig. 2 a The end of the distal stump suture was looped through the proximal stump Krackow locking loop as the pulley. b The bird-beak arthroscopic suture passer was passed subcutaneously through the heel mini-incision over the calcaneous tuberosity and out from the transverse incision. c The ipsilateral Krackow sutures and the contralateral crisscrossed sutures were subcutaneously pulled through the heel incision. $\mathbf{d}$ Illustration of (a)-(c) 

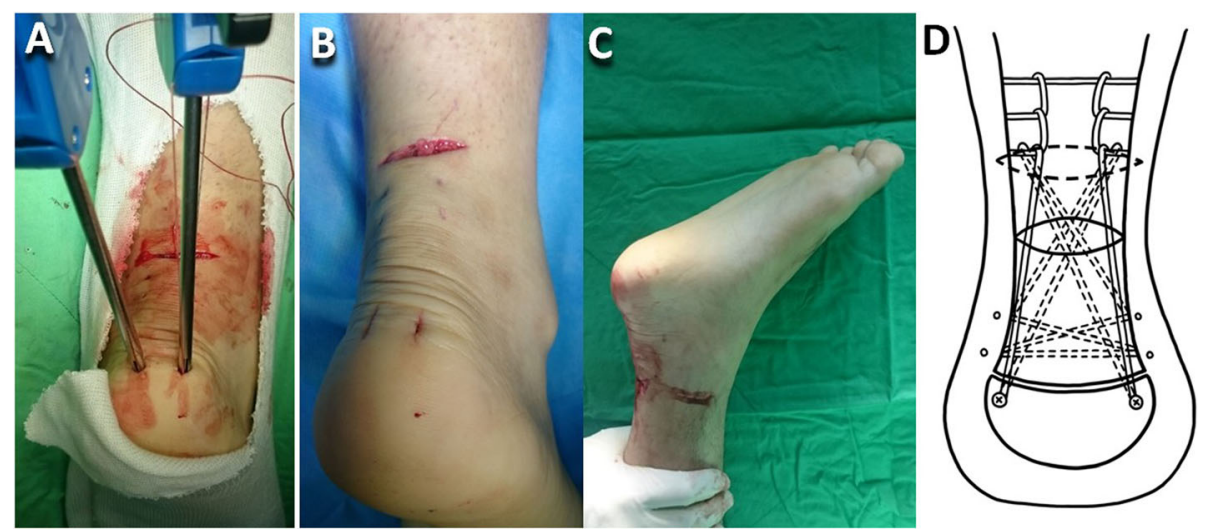

Fig. $\mathbf{3}$ a The sutures were seated at the posterior of the calcaneal tuberosity with two 4.5-mm knotless suture anchors. $\mathbf{b}$ The epitenon was repaired. c The Achilles tendon tension was checked post-operatively with the leg erect. $\mathbf{d}$ Illustration of (a)-(c)

developed, and laboratory studies have reported that tensile strength in the MIS group was comparable with that in the open surgery group [20]. A retrospective series [21] reported that MIS had returned patients to baseline physical activities significantly sooner than open repair did. However, sural nerve injury continues to be the major concern when the Achilles tendon is repaired using MIS [16-18].

A cadaver study [17] reported that the sural nerve crosses the lateral border of the Achilles tendon $8.28-8.96 \mathrm{~cm}$ proximal to the calcaneal tuberosity, which means that surgeons can usually avoid injuring the sural nerve if it is less than $8 \mathrm{~cm}$ proximal to the calcaneal tuberosity. Thus, our modified MIS used Krackow sutures at the proximal stump and percutaneous sutures at the distal stump.
In our series, the mean transverse incision was $6.3 \mathrm{~cm}$ (range, $5.5-7 \mathrm{~cm}$ ) proximal to the calcaneal tuberosity, which corresponded with the common rupture sites and prevented iatrogenic injury of the sural nerve. Furthermore, one study [22] reported that the posterior longitudinal incision was in a less vascularized zone of the skin that covers the Achilles tendon. In our technique, the incisions were transversely proximal to the rupture site to prevent wounds located in less vascularized zones if the incisions are posterolongitudinal. Our patients had no incision complications. The transverse incisions were 2 $\mathrm{cm}$ proximal to the ruptured end; thus, we were able to gently pull out the proximal stump in the knee flexion position. The pulled-out stump with the healthy tendon part was long enough to let us use Krackow sutures,

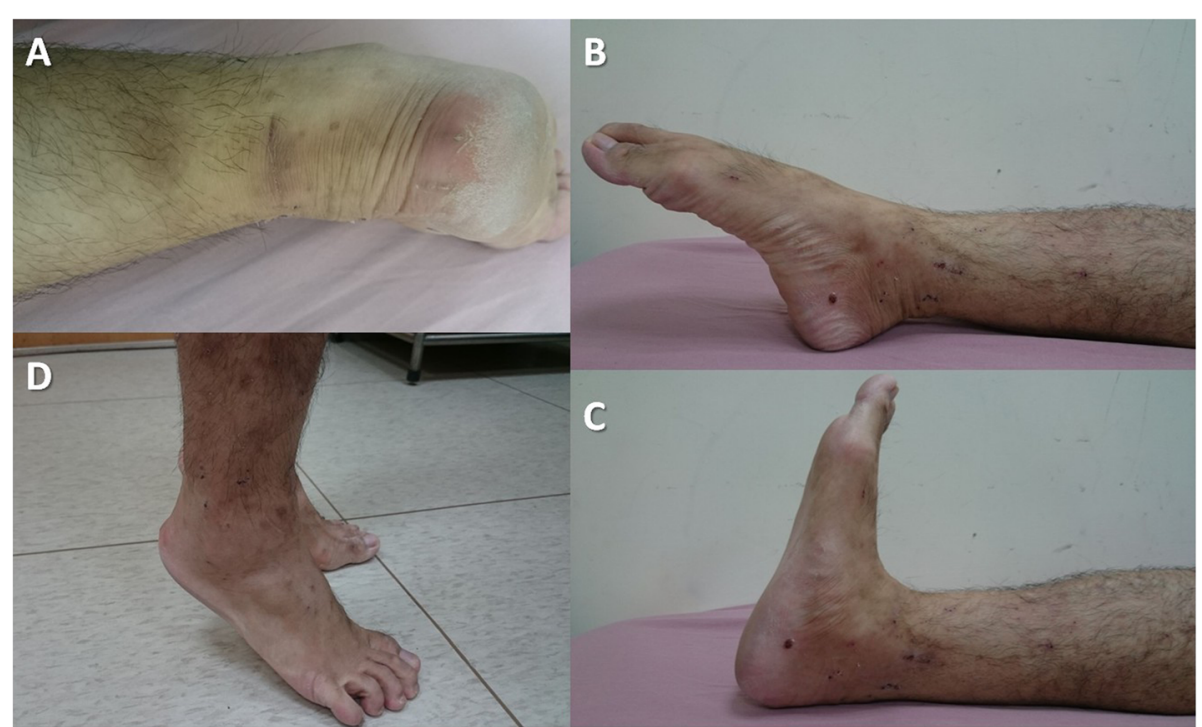

Fig. 4 a The incision scar 4 weeks post-operatively. b Active plantar flexion and c active dorsiflexion 4 weeks post-operatively. $\mathbf{d}$ Heel-raise standing without aid 5 weeks post-operatively 
Table 1 Literature review on post operation rehabilitation protocol and functional outcome in Achilles tendon rupture

\begin{tabular}{|c|c|c|c|c|}
\hline Study & Year & Study type & Surgical method & Rehabilitation protocol \\
\hline $\begin{array}{l}\text { Valkering } \\
\text { KP et al. } \\
{[27]}\end{array}$ & 2016 & $\begin{array}{l}\text { Randomized control } \\
\text { FWB [mobilized full } \\
\text { weight bearing group] } \\
\text { ( } n=27) \text { compared with } \\
\text { IMM [immobilized non- } \\
\text { weight-bearing group] } \\
(n=29)\end{array}$ & $\begin{array}{l}\text { Longitudinal open } \\
\text { incision; end to end } \\
\text { repair with Kessler } \\
\text { sutures }\end{array}$ & $\begin{array}{l}\text { FWB: } \\
\text { 0-2nd week: FWB with } \\
\text { adjustable orthosis and } \\
\text { crutch; } 15-30^{\circ} \text { range of } \\
\text { motion in plantar flexion } \\
\text { 3rd-6th weeks: } 5- \\
45^{\circ} \text { range of motion in } \\
\text { plantar flexion } \\
\text { IMM: } \\
\text { 0-2nd week: non-weight- } \\
\text { bearing with crutch; } \\
\text { ankle immobilized in } \\
30^{\circ} \text { of equinus position. } \\
\text { 3rd-6th weeks: FWB with } \\
\text { crutch and wearing the } \\
\text { heel added orthosis. }\end{array}$ \\
\hline
\end{tabular}

\section{Olsson N 2013 Randomized control} et al. [14]

Sarman H 2016 Retrospective analysis. et al. [24]

Bevoni R et al. [8]
Surgical group

(longitudinal wound incision, end to end repair with a modified Kessler technique) ( $n=$ 43) compared with nonsurgical group $(n=45)$
Semi-invasive internal splinting (SIIS group, $n=$ 24) compared with open end to end repair with Krackow sutures (open group, $n=21$ )

Surgical group: $0-2$ nd week: ankle immobilized in a pneumatic walker brace with heel pads producing a

plantarflexion approximately $20^{\circ}$. FWB with crutch was allowed. 3rd week : Early active range of motion and strength training. Non-surgical group: 0-2nd week: The same as the surgical group. 3rd-8th weeks: Immobilized in the brace for 8 weeks.

Ankle immobilized in $30^{\circ}$ plantar flexion with dorsal splint after operation. No further rehabilitation protocol was available in this article.
Surgical group was significantly superior in the drop counter movement jump and hopping in following 1 year. No significant differences between the groups in symptoms, physical activity level, or quality of life.

\section{Six superficial infections in the surgical group.} One patient in IMM group had traumatic rerupture.

months); no differe

(1)

\begin{tabular}{ll} 
Functional outcome & Complication \\
\hline Improved early ankle & One patient in IMM \\
range of motion (6 & group had traumatic \\
months); no difference & rerupture. \\
in following 1 year &
\end{tabular}


Table 1 Literature review on post operation rehabilitation protocol and functional outcome in Achilles tendon rupture (Continued)

\begin{tabular}{|c|c|c|c|c|c|c|}
\hline Study & Year & Study type & Surgical method & Rehabilitation protocol & Functional outcome & Complication \\
\hline & & & & $\begin{array}{l}\text { boot only, active } \\
\text { dorsiflexion of the ankle } \\
\text { is allowed without } \\
\text { passive dorsiflexion. } \\
4 \text { th-5th weeks: Passive } \\
\text { dorsiflexion is allowed to } \\
\text { neutral. } \\
\text { 6th-7th weeks: Remove } \\
\text { boot } \\
\text { 8th week: Passive } \\
\text { dorsiflexion beyond } \\
\text { neutral. }\end{array}$ & & \\
\hline $\begin{array}{l}\text { Yin L et al. } \\
\text { [10] }\end{array}$ & 2017 & Case series & $\begin{array}{l}\text { Panda rope bridge } \\
\text { technique }(n=11)\end{array}$ & $\begin{array}{l}\text { 0-1st week: Active range } \\
\text { of motion without } \\
\text { weight-bearing. } \\
\text { 2nd-6th weeks: FWB } \\
\text { walking without crutches } \\
\text { while wearing a 30-mm- } \\
\text { height heel, which } \\
\text { decreased } 5 \mathrm{~mm} \text { once a } \\
\text { week. } \\
\text { 7th-8th weeks: muscle } \\
\text { strengthening exercises. } \\
\text { 9th week: advised to take } \\
\text { part in athletic exercises } \\
\text { gradually }\end{array}$ & $\begin{array}{l}\text { The mean AOFAS score } \\
\text { at } 12 \text { months was } 100 \text {. }\end{array}$ & Nil \\
\hline $\begin{array}{l}\text { Current } \\
\text { study }\end{array}$ & 2019 & Case series & $\begin{array}{l}\text { Jigless knotless internal } \\
\text { brace technique }(n=10)\end{array}$ & $\begin{array}{l}\text { 0-1st week: FWB with } \\
\text { crutches and wearing } \\
\text { shoes with an added } \\
\text { heel wedge }(3 \mathrm{~cm}) \text {; non- } \\
\text { weight-bearing range of } \\
\text { motion exercise at least } \\
1 \text { h a day. } \\
\text { 2nd week: walking } \\
\text { without ambulatory aids } \\
\text { was allowed. } \\
\text { 3rd-5th weeks: reducing } \\
\text { the added heel wedge } \\
\text { height by } 1 \mathrm{~cm} \text { per } \\
\text { week. } \\
\text { 5th-6th weeks: heel- } \\
\text { raising exercise } \\
7 \text { th week: exercise as } \\
\text { tolerable }\end{array}$ & $\begin{array}{l}\text { The mean AOFAS score } \\
\text { at } 12 \text { months was } 100 \text {. }\end{array}$ & Nil \\
\hline
\end{tabular}

which are simple, commonly used, and strong enough to permit early post-operative rehabilitation [23].

Two other studies [10, 24] described the internal brace concepts in Achilles tendon repair and reported excellent outcomes. To preserve the proximal stump blood supply, these two studies made additional incisions at the gastrocnemius myotendinous junction. We, however, used Krackow sutures at the healthy tendon and additional soleus muscle. There were no reruptures in our case series; thus, the blood supply was not obstructed for tendon healing. Other internal brace techniques have been reported $[25,26]$, but those studies required specially designed suture jigs, which we did not.

Early ankle range-of-motion improved after early postoperative FWB walking [27]. Another study [28] reported a greater risk of ankle stiffness in the non-weight-bearing group. A recent meta-analysis [29] claimed that early functional rehabilitation improved patient satisfaction and facilitated an earlier return to normal everyday activity after Achilles tendon rupture repair than post-operative immobilization did. Furthermore, there were no significant differences in major complications between the two groups [29]. These findings, which agree with ours, indicate that early stretching and stressing of the repaired tendon improve functional outcomes. Variability in rehabilitation protocols, surgical repair techniques, and adopted functional outcome parameters yielded a variety of differing outcomes and complications (Table 1). However, more studies now emphasize early weight-bearing and ankle range-of-motion after adequate repair [10, 14, 25, 27]. Despite differences in the protocols in the above studies, there are no significant differences between our results. 
The present study appears to be the first to report using a jigless knotless internal brace to repair acute Achilles tendon ruptures. We found, after 1 year of follow-ups, that this simple technique was efficacious.

\section{Limitations}

Our study has limitations. First, our sample was small: only 10 cases. Second, we did not compare our sample with a control group that had been treated using another repair technique. Third, the follow-up duration was short. Fourth, the number of complications might not be realistically representative because the analyzed sample was small. Further studies with larger samples, longer follow-ups, and a control group are needed to confirm our findings.

\section{Conclusion}

Our jigless knotless internal brace technique is simple and was efficacious. Specially designed tools were unnecessary. There were few soft tissue complications. Functional recovery was facilitated because the blood supply to the Achilles tendon was preserved, and because of the strong suture structure. Thus, this technique might be a reliable option for repairing ruptured Achilles tendons.

\section{Abbreviations}

AOFAS scale: American Orthopaedic Foot and Ankle Society scale; FWB: Full weight bearing; MIS: Minimally invasive surgery

\section{Acknowledgements}

We are grateful to Skeleton Materials and Bio-compatibility Core Lab, Research Center of Clinical Medicine, National Cheng Kung University Hospital, for the assistance of this study. We thank the Taiwan National Science Council (grants: MOST 107-2314-B-006-065-MY3, MOST-107-2622-E006-019-CC2, MOST 106-2314-B-006-004, and MOST 108-2314-B-006-011MY2) and National Cheng Kung University Hospital (grants: NCKUH-107-02024 and NCKUH-106-03-030) for funding this work. We are grateful to YingChiu Lin and Yu-Ying Chen for their excellent assistance.

\section{Authors' contributions}

PYK, MTH, and PTW conceived and designed the study. PYK performed the study. PYK, MTH, CLL, IMJ, and PTW analyzed the data. WRS, IMJ, and PTW contributed reagents/materials/analysis tools. PYK and PTW wrote the manuscript. All authors read and approved the final manuscript.

\section{Funding}

Taiwan National Science Council (grants: MOST 107-2314-B-006-065-MY3, MOST-107-2622-E-006-019-CC2, MOST 106-2314-B-006-004, and MOST 1082314-B-006-011-MY2) and National Cheng Kung University Hospital (grants: NCKUH-107-02-024, NCKUH-106-03-030)

\section{Availability of data and materials}

The datasets used and analyzed during the current study are available from the corresponding author on reasonable request.

\section{Ethics approval and consent to participate}

All procedures were approved by our hospital's Institutional Review Board. IRB: Human Experiment and Ethics Committee (HEEC) of National Cheng Kung University Hospital (NCKUH)

IRB No.: B-ER-106-256
Consent for publication

Not applicable

\section{Competing interests}

The authors declare that they have no competing interests.

\section{Author details}

${ }^{1}$ Department of Biomedical Engineering, National Cheng Kung University, Tainan, Taiwan. ${ }^{2}$ Department of Orthopedics, National Cheng Kung University Hospital Dou-Liu Branch, College of Medicine, National Cheng Kung University, Yunlin, Taiwan. ${ }^{3}$ Department of Orthopedics, National Cheng Kung University Hospital, College of Medicine, National Cheng Kung University, Tainan, Taiwan. ${ }^{4}$ Department of Orthopedics, College of Medicine, National Cheng Kung University, 1 University Road, East District, Tainan City 701, Taiwan. ${ }^{5}$ Department of Orthopedics, E-Da Hospital, Kaohsiung, Taiwan. ${ }^{6}$ School of Medicine, College of Medicine, I-Shou University, Kaohsiung, Taiwan. ${ }^{7}$ Medical Device Innovation Center, National Cheng Kung University, Tainan, Taiwan.

Received: 22 July 2019 Accepted: 13 November 2019

Published online: 05 December 2019

\section{References}

1. Suchak AA, Bostick G, Reid D, et al. The incidence of Achilles tendon ruptures in Edmonton, Canada. Foot Ankle Int. 2005;26(11):932-6.

2. Lantto I, Heikkinen J, Flinkkila T, et al. Epidemiology of Achilles tendon ruptures: increasing incidence over a 33-year period. Scand J Med Sci Sports. 2015;25(1):e133-8.

3. Ganestam A, Kallemose T, Troelsen A, et al. Increasing incidence of acute Achilles tendon rupture and a noticeable decline in surgical treatment from 1994 to 2013. A nationwide registry study of 33,160 patients. Knee Surg Sports Traumatol Arthrosc. 2016:24(12):3730-7.

4. Huttunen $T$, Kannus $P$, Rolf $C$, et al. Acute Achilles tendon ruptures: incidence of injury and surgery in Sweden between 2001 and 2012. Am J Sports Med. 2014;42(10):2419-23.

5. Khan RJ, Fick D, Keogh A, et al. Treatment of acute Achilles tendon ruptures. A meta-analysis of randomized, controlled trials. J Bone Joint Surg Am. 2005;87(10):2202-10.

6. Carmont MR, Rossi R, Scheffler $\mathrm{S}$, et al. Percutaneous \& mini invasive Achilles tendon repair. Sports Med Arthrosc Rehabil Ther Technol. 2011;3:28.

7. Uquillas CA, Guss MS, Ryan DJ, et al. Everything Achilles: knowledge update and current concepts in management: AAOS Exhibit Selection. J Bone Joint Surg Am. 2015:97(14):1187-95.

8. Bevoni R, Angelini A, D'Apote $G$, et al. Long term results of acute Achilles repair with triple-bundle technique and early rehabilitation protocol. Injury. 2014;45(8):1268-74.

9. Avina Valencia JA, Guillen Alcala MA. Repair of acute Achilles tendon rupture. Comparative study of two surgical techniques. Acta Ortop Mex. 2009;23(3):125-9.

10. Yin $L, W u Y$, Ren $C$, et al. Treatment of acute Achilles tendon rupture with the panda rope bridge technique. Injury. 2018;49(3):726-9.

11. Keating JF, Will EM. Operative versus non-operative treatment of acute rupture of tendo Achillis: a prospective randomised evaluation of functional outcome. J Bone Joint Surg Br. 2011:93(8):1071-8.

12. Huang J, Wang $C, M a X$, et al. Rehabilitation regimen after surgical treatment of acute Achilles tendon ruptures: a systematic review with metaanalysis. Am J Sports Med. 2015;43(4):1008-16

13. Soroceanu A, Sidhwa F, Aarabi S, et al. Surgical versus nonsurgical treatment of acute Achilles tendon rupture: a meta-analysis of randomized trials. J Bone Joint Surg Am. 2012;94(23):2136-43.

14. Olsson N, Silbernagel KG, Eriksson Bl, et al. Stable surgical repair with accelerated rehabilitation versus nonsurgical treatment for acute Achilles tendon ruptures: a randomized controlled study. Am J Sports Med. 2013; 41(12):2867-76.

15. McMahon SE, Smith TO, Hing CB. A meta-analysis of randomised controlled trials comparing conventional to minimally invasive approaches for repair of an Achilles tendon rupture. Foot Ankle Surg. 2011;17(4):211-7.

16. Paavola M, Orava S, Leppilahti J, et al. Chronic Achilles tendon overuse injury: complications after surgical treatment. An analysis of 432 consecutive patients. Am J Sports Med. 2000;28(1):77-82. 
17. Citak M, Knobloch $K$, Albrecht $K$, et al. Anatomy of the sural nerve in a computer-assisted model: implications for surgical minimal-invasive Achilles tendon repair. Br J Sports Med. 2007;41(7):456-8 discussion 8.

18. Klein W, Lang DM, Saleh M. The use of the Ma-Griffith technique for percutaneous repair of fresh ruptured tendo Achillis. Chir Organi Mov. 1991; 76(3):223-8.

19. Blackmon JA, Atsas S, Clarkson MJ, et al. Locating the sural nerve during calcaneal (Achilles) tendon repair with confidence: a cadaveric study with clinical applications. J Foot Ankle Surg. 2013;52(1):42-7.

20. Ismail M, Karim A, Shulman $\mathrm{R}$, et al. The Achillon Achilles tendon repair: is it strong enough? Foot Ankle Int. 2008;29(8):808-13.

21. Hsu AR, Jones CP, Cohen BE, et al. Clinical outcomes and complications of percutaneous Achilles repair system versus open technique for acute Achilles tendon ruptures. Foot Ankle Int. 2015;36(11):1279-86.

22. Yepes $H$, Tang $M$, Geddes $C$, et al. Digital vascular mapping of the integument about the Achilles tendon. J Bone Joint Surg Am. 2010;92(5): $1215-20$.

23. Maffulli $\mathrm{N}$, Longo UG, Oliva $\mathrm{F}$, et al. Minimally invasive surgery of the Achilles tendon. Orthop Clin North Am. 2009;40(4):491-8 viii-ix.

24. Sarman H, Muezzinoglu US, Memisoglu K, et al. Comparison of semiinvasive "internal splinting" and open suturing techniques in Achilles tendon rupture surgery. J Foot Ankle Surg. 2016;55(5):965-70.

25. McWilliam JR, Mackay G. The internal brace for midsubstance Achilles ruptures. Foot Ankle Int. 2016;37(7):794-800.

26. Liechti DJ, Moatshe G, Backus JD, et al. A percutaneous knotless technique for acute Achilles tendon ruptures. Arthrosc Tech. 2018;7(2):e171-e8.

27. Valkering KP, Aufwerber S, Ranuccio F, et al. Functional weight-bearing mobilization after Achilles tendon rupture enhances early healing response: a single-blinded randomized controlled trial. Knee Surg Sports Traumatol Arthrosc. 2017;25(6):1807-16.

28. Barfod KW, Bencke J, Lauridsen HB, et al. Nonoperative, dynamic treatment of acute Achilles tendon rupture: influence of early weightbearing on biomechanical properties of the plantar flexor muscle-tendon complex-a blinded, randomized, controlled trial. J Foot Ankle Surg. 2015;54(2):220-6.

29. McCormack R, Bovard J. Early functional rehabilitation or cast immobilisation for the postoperative management of acute Achilles tendon rupture? A systematic review and meta-analysis of randomised controlled trials. $\mathrm{Br} J$ Sports Med. 2015;49(20):1329-35.

\section{Publisher's Note}

Springer Nature remains neutral with regard to jurisdictional claims in published maps and institutional affiliations.

Ready to submit your research? Choose BMC and benefit from:

- fast, convenient online submission

- thorough peer review by experienced researchers in your field

- rapid publication on acceptance

- support for research data, including large and complex data types

- gold Open Access which fosters wider collaboration and increased citations

- maximum visibility for your research: over $100 \mathrm{M}$ website views per year

At $\mathrm{BMC}$, research is always in progress.

Learn more biomedcentral.com/submissions 